\title{
Autoeficácia e nível de ansiedade em atletas jovens do atletismo paranaense
}

\author{
Self-efficacy and level of anxiety in young track and field \\ athletes from Paraná
}

Lenamar Fiorese Vieira

Cesar Luis Teixeira

José Luis Lopes Vieira ${ }^{1}$ Albertino Oliveira Filho ${ }^{2}$

\footnotetext{
1 Universidade Estadual de Maringá. Programa de Pós-Graduação Associado em Educação Física. Maringá, PR. Brasil.

2 Universidade Estadual de Maringá. Maringá, PR. Brasil.
}

Recebido em 13/08/10 Revisado em 17/11/10 Aprovado em 03/01/11
Resumo - Este estudo analisou a autoeficácia em relação ao nível de ansiedade de atletas do atletismo no Estado do Paraná. A amostra constituiu-se de 75 atletas de idade média de 16,76 anos e de ambos os sexos. Como instrumento foram utilizados o Inventário de Ansiedade Estado Competitiva (CSAI-2), e um questionário estruturado para identificar a autoeficácia. Para análise estatística, foram utilizados os testes Kolmogorov-Smirnov, Mann-Whitney e Kruskall-Wallis. Os resultados indicaram que não houve diferença significativa nos níveis de ansiedade quanto ao gênero; para as mulheres, não foram encontradas diferenças significativas entre expectativa de resultado e níveis de ansiedade; os homens que obtiveram resultado superior ao esperado apresentaram maior nível de ansiedade. Concluiu-se que os atletas com altas expectativas de resultado apresentaram níveis maiores de ansiedade cognitiva e somática e de autoconfiança.

Palavras-chave: Ansiedade; Autoeficácia; Esporte.

Abstract - This study analyzed self-efficacy in relation to anxiety level in young track and field athletes from Paraná state. The sample consisted of 75 athletes of both genders with a mean age of 16.76 years. The Competitive State Anxiety Inventory (CSAI-2) was used to assess competitive state anxiety and a structured questionnaire was used to identify self-efficacy. The Kolmogorov-Smirnov, Mann-Whitney, and Kruskal-Wallis tests were used for statistical analysis. The results showed no significant difference in anxiety levels between genders. For women, no significant differences were observed between outcome expectancy and anxiety level. Men who achieved a better result than expected presented a higher anxiety level. In conclusion, athletes with high outcome expectations show higher levels of cognitive and somatic anxiety and self-confidence. Key words: Self-efficacy; Youth; Sports; Anxiety. 


\section{INTRODUÇÃO}

A autoeficácia refere-se às crenças que a pessoa tem acerca da sua capacidade para organizar e executar ações requeridas para alcançar determinados padrões de desempenho ${ }^{1}$. A percepção da autoeficácia não é relativa ao número de habilidades que temos, mas ao modo como julgamos o que fazer com o que temos, sob uma variedade de circunstâncias².

Desta forma, existem dois componentes da autoeficácia ${ }^{3}$ : uma expectativa de eficácia e uma expectativa de resultado. A expectativa de eficácia é a convicção que o próprio atleta tem de possuir capacidade para gerar o resultado, e a expectativa de resultado se refere à crença do atleta de que um dado comportamento levará a um resultado específico.

Neste sentido, as crenças da autoeficácia ajudam o atleta a fazer as escolhas das atividades e os meios para atingir suas metas, assim como o esforço a ser despendido, a persistência na tarefa e as reações emocionais quando são confrontadas com os obstáculos. Assim, a confiança do atleta em sua capacidade para desempenhar com sucesso determinada tarefa ou conjunto de tarefas ajuda a determinar se ele irá iniciar, perseverar e ser bem-sucedido em determinados desempenhos ${ }^{4}$. $\mathrm{O}$ desempenho bem-sucedido tenderá a aumentar as crenças do self e dos resultados, fortalecendo os interesses e os objetivos do atleta nesse domínio de desempenho 5 .

A lacuna encontrada nos estudos de autoeficácia está na análise do nível de ansiedade-estado e expectativa de resultado no contexto atletismo, entendendo-se que, independentemente da modalidade que pratica, o atleta é um exemplo de pessoa que vive constantemente em situações ansiógenas, por ser exposto a comentários críticos tanto pelos meios de comunicação quanto pela torcida, por seus pares e pelo próprio treinador ${ }^{6}$. Nos momentos antecedentes à competição, o atleta prevê as oportunidades, os riscos e as consequências, o que origina, frequentemente, medos e temores, podendo se manifestar de forma cognitiva, física, motora e emocional.

Um estudo realizado com tenistas observou que o efeito da percepção da autoeficácia no desempenho esportivo não substitui o talento físico, podendo ser considerada uma codeterminante do desempenho. Ela é uma mistura do dom físico e das capacidades desenvolvidas experimentalmente, ressaltando-se que o que os atletas fazem com o que sabem e com o que têm é, parcialmente, determinado por sua percepção de eficácia pessoal? ${ }^{7}$.

Jackson et al. ${ }^{8}$ acrescentam que experiências de sucesso numa dada tarefa aumentam as estimativas de eficácia, enquanto os fracassos repetidos tendem a diminuí-las. Ainda, atletas com habilidades semelhantes, mas com diferentes níveis de segurança em si mesmos terão desempenhos diferentes ${ }^{1}$.

Neste contexto, a interpretação que um indivíduo faz dos sintomas de ansiedade é importante para o entendimento da relação ansiedade-desempenho. As pessoas podem considerar os sintomas de ansiedade tanto positivos e úteis para o desempenho (facilitadores) como negativos e prejudiciais ao desempenho (debilitantes), que levam o indivíduo a uma ação menos significativa em tarefas de várias espécies?.

Em face dessas considerações, o objetivo do estudo foi analisar a expectativa de resultado em relação ao nível de ansiedade de atletas da modalidade de Atletismo do Estado do Paraná. Especificamente, este estudo buscou identificar o nível da ansiedade-estado pré-competitiva dos atletas; diagnosticar a expectativa de resultado dos atletas para a competição, verificar o resultado alcançado na competição e comparar o nível de ansiedade-estado em relação ao resultado alcançado na competição.

Hipóteses conceituais foram estabelecidas para o presente estudo: (1) atletas do gênero feminino apresentam níveis de ansiedade maior que os do gênero masculino; (2) indivíduos com alta expectativa de resultado apresentariam escores maiores de ansiedade bem como melhores desempenhos; (3) indivíduos com baixa expectativa de resultado apresentariam maiores escores de ansiedade bem como baixos desempenhos esportivos.

\section{PROCEDIMENTOS METODOLÓGICOS}

A pesquisa está integrada ao projeto institucional sob parecer do Comitê de Ética em Pesquisa com Seres Humanos $n^{\circ}$ 175/2007. A amostra, do tipo intencional, constituiu-se de 75 atletas de idades entre 14 e 19 anos, sendo 47 masculinos e 28 femininos, todos da Federação Paranaense de Atletismo.

Para identificar a expectativa de resultado, os atletas foram questionados sobre qual era a expectativa de resultado (convicção) de cada um para aquela competição e no final da competição, foi verificado cada resultado conquistado (acima do esperado, abaixo do esperado e esperado).

Para identificar a ansiedade-estado pré-competitiva foi utilizada a escala de ansiedade-estado pré-competitiva específica para esporte (CSAI-2), desenvolvida por Martens et al. ${ }^{10}$ e validada para língua portuguesa por Santos e Serpa ${ }^{11}$. A escala 
divide ansiedade em três componentes: cognitivo, somático e um componente relacionado à autoconfiança. O CSAI-2 é composto de 27 questões com escala Likert nas quais o sujeito opta por $1=$ nada, 2 = um pouco, 3 = moderadamente e $4=$ muito. Na pontuação, que varia de 9 a 36 pontos, 9 indica baixa ansiedade (confiança) e 36 indica alta ansiedade.

A coleta de dados foi realizada na competição estadual de 2008. Os atletas respondiam individualmente ao questionário CSAI-2 antes de participar da sua prova específica e identificavam a sua expectativa de resultado, tempo de treino (em anos), volume de treino semanal (em horas) e quantidade de medalhas conquistadas do início do ano até aquele momento, dados que eram registrados em sua ficha individual.

Os dados foram organizados no programa excel ${ }^{\circledR}$ for Windows. $O$ tratamento estatístico foi realizado por meio do Programa SPSS 13 for Windows. Foram utilizados os recursos da estatística descritiva (Frequência e percentual). A distribuição de normalidade dos dados foi verificada por meio do teste de Kolmogorov-Smirnov.

Os dados não apresentaram uma distribuição normal, desta forma a verificação de diferenças entre as médias foi feita por meio dos testes $U$ de Mann-Whitney para grupos independentes e de Kruskall-Wallis para comparação de mais de dois grupos independentes.

\section{RESULTADOS}

Na tabela 1, apresentam-se as médias de idade e tempo de treinamento, as quais não diferem entre os gêneros $(\mathrm{p}<0,05)$. Os atletas são jovens $(16,8$ anos) e têm pouco tempo de prática $(3,6)$, apesar das conquistas já obtidas.

Tabela 1. Características do perfil dos atletas de atletismo do Paraná.

\begin{tabular}{|c|c|c|c|c|c|}
\hline \multirow{4}{*}{ Percepção } & \multicolumn{4}{|c|}{ Gênero } & \multirow{4}{*}{$\mathrm{x}$} \\
\hline & \multicolumn{2}{|c|}{ Masculino } & \multicolumn{2}{|c|}{ Feminino } & \\
\hline & $\mathrm{n}$ & $\%$ & $\mathrm{n}$ & $\%$ & \\
\hline & 47 & 62,67 & 28 & 37,33 & \\
\hline Idade (anos) & \multicolumn{2}{|c|}{$16,85 \pm 1,19$} & \multicolumn{2}{|c|}{$16,60 \pm 1,42$} & 16,76 anos \\
\hline Treino (anos) & \multicolumn{2}{|c|}{$3,39 \pm 2,12$} & \multicolumn{2}{|c|}{$3,95 \pm 2,45$} & 3,6 anos \\
\hline Treino (horas) & \multicolumn{2}{|c|}{$14,98 \pm 5,94$} & \multicolumn{2}{|c|}{$12,83 \pm 3,77$} & 14,36 horas \\
\hline Medalhas & \multicolumn{2}{|c|}{$2,72 \pm 2,92$} & \multicolumn{2}{|c|}{$3,82 \pm 3,97$} & 3,2 \\
\hline
\end{tabular}

Na expectativa de resultado dos atletas (tabela 2), verifica-se maior concentração dos atletas, com expectativa de eficácia para alcançar o primeiro lugar. No grupo masculino (58\%) e no feminino (64\%) dos atletas tinham expectativas de vencer (conquistar o $1^{\circ}$ lugar) suas provas.

A tabela 3 evidencia que não houve diferenças estatisticamente significativas dos níveis de ansiedade. Na comparação da expectativa de resultado com o nível de ansiedade dos atletas juvenis, percebe-se (tabela 4) que atletas que tinham expectativas altas de desempenho apresentavam níveis altos de ansiedade em todas as subescalas, diferença que é mais evidente na ansiedade somática, quando os atletas relatam sentir alterações fisiológicas no corpo. Por outro lado, percebe-se que os atletas que conquistaram o resultado esperado tiveram um nível moderado de ansiedade em todas as subescalas.

Na tabela 5, verificam-se diferenças significativas entre os que atingiram resultados melhores que o esperado e os que atingiram os resultados

Tabela 2. Expectativa de resultado quanto ao gênero e resultado competitivo dos atletas.

\begin{tabular}{|c|c|c|c|c|c|c|}
\hline \multirow{2}{*}{ Genêro } & \multirow{2}{*}{ Autoeficácia } & \multicolumn{5}{|c|}{ Resultado da competição } \\
\hline & & $1^{\circ}$ lugar & $2^{\circ}$ Lugar & $3^{\circ}$ lugar & 4-8 ${ }^{\circ}$ Lugares & Total \\
\hline \multirow{2}{*}{ Masc } & Expectativa & $25(58 \%)$ & $04(9 \%)$ & $07(16 \%)$ & $07(16 \%)$ & $43(100 \%)$ \\
\hline & Alcançaram & $12(28 \%)$ & $02(4 \%)$ & $01(2 \%)$ & $01(2 \%)$ & $16(36 \%)$ \\
\hline \multirow{2}{*}{ Fem } & Expectativa & $18(64 \%)$ & $05(18 \%)$ & $01(4 \%)$ & $04(14 \%)$ & $28(100 \%)$ \\
\hline & Alcançaram & 07 (25\%) & 03 (11\%) & $01(4 \%)$ & 02 (7\%) & 13 (47\%) \\
\hline
\end{tabular}

Tabela 3. Comparação dos níveis de ansiedade entre os gêneros dos atletas do atletismo.

\begin{tabular}{lccccc}
\hline Ansiedade & $\mathrm{n}$ & $\%$ & Cognitiva & Somática & Autoconfiança \\
\hline Gênero & & & & & \\
Masculino & 43 & 62,67 & $20,31 \pm 5,75$ & $17,51 \pm 4,57$ & $20,46 \pm 4,96$ \\
Feminino & 28 & 37,33 & $19,89 \pm 4,25$ & $18,32 \pm 4,38$ & $21,82 \pm 3,75$ \\
Geral & 71 & 100 & $20,16 \pm 5,21$ & $17,81 \pm 4,49$ & $20,97 \pm 4,57$ \\
\hline
\end{tabular}


Tabela 4. Comparação da expectativa de resultado e o nível da ansiedade dos atletas de atletismo.

\begin{tabular}{|c|c|c|c|c|c|c|c|}
\hline \multirow{4}{*}{ Ansiedade } & \multicolumn{6}{|c|}{ Percepção de autoeficácia } & \multirow{4}{*}{$P$} \\
\hline & \multicolumn{2}{|c|}{ Abaixo } & \multicolumn{2}{|c|}{ Esperado } & \multicolumn{2}{|c|}{ Acima } & \\
\hline & $\mathrm{n}$ & $\%$ & $\mathrm{n}$ & $\%$ & $\mathrm{n}$ & $\%$ & \\
\hline & 41 & 54,7 & 30 & 40,0 & 04 & 5,3 & \\
\hline Cognitiva & \multicolumn{2}{|c|}{$19,97 \pm 4,89$} & \multicolumn{2}{|c|}{$19,53 \pm 4,86$} & \multicolumn{2}{|c|}{$26,75 \pm 7,80$} & 0,129 \\
\hline Somática & \multicolumn{2}{|c|}{$17,73 \pm 4,04$} & \multicolumn{2}{|c|}{$16,86 \pm 3,59$} & \multicolumn{2}{|c|}{$25,75 \pm 7,80$} & $0,035^{*}$ \\
\hline Auto-confiança & \multicolumn{2}{|c|}{$21,12 \pm 4,30$} & \multicolumn{2}{|c|}{$20,16 \pm 4,49$} & \multicolumn{2}{|c|}{$25,50 \pm 6,24$} & 0,188 \\
\hline
\end{tabular}

$(\mathrm{p}<0,05)$

Tabela 5. Comparação entre o resultado das competições e o nível de ansiedade dos atletas de atletismo.

\begin{tabular}{lccc}
\hline & & Ansiedade & \\
\cline { 2 - 4 } Resultados & Cognitiva & Somática & Autoconfiança \\
\hline Acima x Esperado & $0,039^{*}$ & $0,013^{*}$ & 0,086 \\
Acima x Abaixo & 0,072 & $0,024^{*}$ & 0,133 \\
Esperado x Abaixo & 0,661 & 0,362 & 0,417 \\
\hline
\end{tabular}

$(\mathrm{p}<0,05)$

esperados, para as variáveis ansiedade cognitiva $(\mathrm{p}=0,039)$ e ansiedade somática $(\mathrm{p}=0,013)$, demonstrando que os atletas que conquistavam resultados acima das suas expectativas estavam em um nível de ansiedade alto.

Entre os atletas que obtiveram resultado acima do esperado e abaixo do esperado para a variável ansiedade somática $(\mathrm{p}=0,024)$, ficou evidenciado que aqueles da primeira categoria estavam em nível de ansiedade alta, o que pode ter interferido nas crenças e consequentemente, na sua percepção de expectativa de resultado, enquanto os da segunda categoria estavam em nível moderado de ansiedade.

\section{DISCUSSÃO}

Para a expectativa de resultado quanto ao gênero e ao resultado competitivo, $28 \%$ dos atletas do gênero masculino e $25 \%$ dos do gênero feminino tiveram confirmadas as suas expectativas (Tabela 2). Para a expectativa de medalha de prata, as atletas atingiram $11 \%$ de suas metas, enquanto os atletas (gênero masculino) atingiram apenas $4 \%$. No total, tiveram confirmadas as suas expectativas de eficácia 47\% das mulheres e $36 \%$ dos homens. Neste sentido, os resultados evidenciam que as mulheres apresentam expectativas de resultado mais realistas para seus desempenhos.

Na comparação dos níveis de ansiedade entre os gêneros dos atletas do Atletismo (Tabela 3,) os dados vão de encontro às pesquisas realizadas na canoagem $^{12}$, em que o gênero feminino apresentava um nível de ansiedade somática e cognitiva maior que o gênero masculino e que esta diferença influenciava de modo significativo no desempenho das atletas, ou seja, os homens, ao manifestarem um menor índice de ansiedade somática e cognitiva, obtiveram um melhor desempenho do que as mulheres nas diferentes provas do esporte.

Também em estudo com judocas ${ }^{13}$, encontraram-se resultados de um maior nível de ansiedade-traço pré-competitiva em atletas do gênero feminino quando comparados com os do gênero masculino. Diferenças significativas foram encontradas ${ }^{14}$ nos níveis de ansiedade-traço entre atletas de diferentes gêneros no atletismo infantil (14-16), tendo o gênero feminino apresentado os maiores níveis, destacando-se que a diferença se acentuava com o aumento da idade.

Estudo realizado com atletas de ambos os gêneros na modalidade de voleibol ${ }^{15}$ não encontraram resultados estatisticamente significantes entre os gêneros em nenhuma subescala, fato que se assemelha ao que se constatou no presente estudo. Cumpre ressaltar que, com a evolução do esporte e o investimento na preparação mental dos atletas, as mulheres estão controlando melhor suas emoções e atingindo as suas expectativas de autoeficácia.

Na comparação da expectativa de resultado com o nível da ansiedade dos atletas (Tabela 4), este estudo permite inferir que os atletas que apresentaram percepção de eficácia com convicção da sua capacidade se encontravam em nível de ativação moderado, sendo este o ideal para a modalidade de atletismo, que necessita de concentração, precisão e sincronia de movimentos. Estudo anterior ${ }^{14} \mathrm{com}$ atletas juvenis de atletismo também encontrou níveis de ansiedade considerados moderados. 
Na comparação entre os resultados das competições e o nível de ansiedade dos atletas (Tabela 5), os resultados destacam a necessidade de investigação nos atletas que criam expectativas irrealistas sobre seu desempenho, fortalecendo crenças que podem afetar o julgamento de valor de suas capacidades.

As diferenças nas subescalas cognitiva e somática da ansiedade foram observadas nos atletas que alcançaram as suas metas (o esperado). Estudos ${ }^{16}$ demonstram que a situação competitiva implica sempre um aumento dos níveis de ansiedade e do estresse, cujos efeitos alteram o rendimento competitivo, normalmente, prejudicando o sucesso desportivo. Embora, porém, em certos casos, atletas apresentem elevadas competências psicológicas, a ansiedade e o estresse pré-competitivo podem ter efeitos positivos na prestação desportiva, o que pode ter sido o caso destes atletas.

Em relação aos atletas que não atingiram suas expectativas (acima ou abaixo), percebe-se que os atletas somatizam mais a expectativa de resultado $(\mathrm{p} \leq 0,05)$. Essas diferenças evidenciam que os atletas que têm expectativas de eficácia acima de suas capacidades de desempenho apresentaram, também, maiores níveis de ansiedade.

Estes resultados vão ao encontro da teoria de Bandura ${ }^{17}$, segundo a qual bons resultados estão relacionados a uma real percepção de autoeficácia e consequentemente, a um nível de ansiedade adequado.

Estados somáticos e emocionais como a ansiedade, o estresse, a excitação e os estados de humor também proporcionam informações sobre as crenças de autoeficácia ${ }^{18}$. As pessoas podem avaliar o seu grau de confiança por seu estado fisiológico enquanto pensam em uma determinada ação. Reações emocionais fortes a uma tarefa fornecem pistas sobre a previsão de sucesso ou fracasso. Quando as pessoas têm pensamentos negativos e temores sobre sua capacidade (ansiedade cognitiva), as reações afetivas podem reduzir as percepções de autoeficácia e desencadear mais estresse e agitação.

Estes resultados corroboram o argumento de que nem toda ansiedade é prejudicial ${ }^{13}$. O bom desempenho requer um nível de ansiedade ótimo, e quando isto acontece o atleta está psicologicamente em controle. Assim, mais importante do que simplesmente saber quanto de ansiedade um atleta tem é saber qual é o nível de ansiedade ótimo que o faz ter melhores resultados esportivos e expectativas de resultados realistas ${ }^{19}$. Não ter investigado o nível ótimo de ansiedade de cada atleta e a expectativa de eficácia foram limitações do estudo.

\section{CONCLUSÃO}

As hipóteses conceituais do estudo de que atletas do gênero feminino apresentam níveis de ansiedade maiores que os do gênero masculino e de que indivíduos com baixa expectativa de resultado apresentariam maiores escores de ansiedade e baixos desempenhos esportivos não foram confirmadas. Por outro lado, foi confirmada a hipótese de que indivíduos com alta expectativa de resultados apresentariam escores maiores de ansiedade bem como melhores desempenhos.

Comparando-se os resultados competitivos (esperado, abaixo ou acima), encontraram-se diferenças significativas para as ansiedades somática e cognitiva entre resultado abaixo versus resultado acima e entre resultado esperado versus resultado acima do esperado, o que demonstra que as crenças cognitivas dos atletas do atletismo têm interferência na autoeficácia (resultados das competições), levando, na maioria das vezes, à somatização dos sucessos ou insucessos.

\section{REFERÊNCIAS BIBLIOGRÁFICAS}

1. Bandura A. Self-efficacy: The exercise of control. New York: W. H. Freeman and Company; 1997.

2. Bandura A, Jourden FJ. Self-Regulatory Mechanisms Governing the Impact of Social Comparison on Complex Decision Making. J Pers Soc Psychol 1991; 60(6):941-51.

3. Hall C, Lindzey G, Campbell JB. Teorias da personalidade. Porto Alegre: Artmed; 2000.

4. Lent RW, Hackett G, Brown SD. Una perspectiva Social Cognitiva de la transición entre la escuela y el trabajo. Evaluar 2004;4:1-7.

5. Azzi RG, Polydoro SAJ. Autoeficácia em diferentes contextos. Campinas: Alínea; 2006.

6. Cratty BJ. Psicologia do esporte. São Paulo: Prentice-hall do Brasil; 1984.

7. Moreno RM. Autoeficácia de tenistas e desempenho esportivo: perspectivas da psicologia do esporte. Dissertação (Mestrado em Educação Física). Universidade Estadual Paulista, Bauru, Brasil; 2007.

8. Jackson MA, Potere JC, Brobst KA. Are Success Learning Experiences and Self-Efficacy Beliefs Associated With Occupational Interests and Aspirations of At-Risk Urban Youth? J Car Ass 2006;14:333-3.

9. Weinberg RS, Gould D. Fundamentos da Psicologia do Esporte e do Exercício. Porto Alegre: Artmed; 2001.

10. Martens R, Vealey R, Burton D. Competitive anxiety in Sport. Champaign: Human Kinetics; 1990.

11. Santos A, Serpa S. Ansiedade competitiva. Relação treinador atleta - Estudo da influência do treinador em atletas de tênis. Tese (Doutorado em Psicologia). Universidade Técnica de Lisboa, Lisboa, Portugal; 1991. 
12. Lavoura TN, Botura HML, Machado AA. Estudo da ansiedade e as diferenças entre os gêneros em um esporte de aventura competitivo. Rev Bras Educ Fís Esporte, Lazer e Dança 2006; 1(3):74-81.

13. Detanico D, Santos SG. Variáveis influenciando e sendo influenciadas pela ansiedade traço pré-competitiva: um estudo com judocas. Revista Digital 2005; 10(90); Available from:< http://www.efdeportes.com> [2009 jun 15].

14. De Rose Junior D, Vasconcellos EG. Ansiedade traço competitiva e atletismo: um estudo com atletas infanto-juvenis. Rev Paul Educ Fís São Paulo 1997; 11(2):148-54.

15. Seeley G, Storey J, Wagner D, Walker C, Watts K. Anxiety levels and gender differences in social volleyball players before and during competition in an Australian setting. 2008; Available from: <http://www.geocities. com/CollegePark/5686/su99 p5.htm> [2008 jul 15].

16. Hall HK, Kerr AW, Matthews J. Precompetitive anxiety in sport: the contribution of achievement goals and perfectionism. J Sport Exerc Psychol 1998;20:194-217.
17. Bandura, A. Self-efficacy. In: Ramachaudran VS, organizador. Encyclopedia of human behavior. New York: Academic Press; 1994. p.71-81.

18. Pajares F, Olaz F. Teoria social cognitiva e autoeficácia: uma visão geral. In Bandura A, Azzi RG, Polydoro S, organizadores. Teoria Social Cognitiva: conceitos básicos. Porto Alegre: ArtMed; 2008. p.115-160.

19. Brandão MRF. Psicologia Esportiva: ansiedade em atletas. Rev Mov 1995; 5(1):24-7.

Endereço para correspondência

Lenamar Fiorese Vieira

Av. Neo Alves Martins, 1886, Apto. 151

Centro, Maringá, Paraná

Email: lfvieira@uem.br 